\title{
Trypanosoma cruzi: Morphological and Microtubular Changes Related to Epimastigote Differentiation under Hyperosmotic Stress
}

\author{
Alba Marina Gimenez ${ }^{1 *}$ and Estela E. Machado ${ }^{1}$ \\ ${ }^{1}$ Department of Molecular Biology, Faculty of Exact Sciences, Physical-Chemical and Natural Sciences, National University of Río Cuarto, Río Cuarto (X5800) \\ Córdoba, Argentina
}

Received: November 28, 2016; Accepted: December 25, 2016; Published: January 03, 2017

*Corresponding author: Alba Marina Gimenez, Department of Molecular Biology, Faculty of Exact Sciences, Physical-Chemical and Natural Sciences, National University of Río Cuarto, Río Cuarto (X5800) Córdoba,Argentina, E-mail: lbmrngmz@gmail.com

Estela E. Machado, Department of Molecular Biology, Faculty of Exact Sciences, Physical-Chemical and Natural Sciences, National University of Río Cuarto, Río Cuarto (X5800) Córdoba, Argentina, E-mail: estelaemachado@gmail.com

\begin{abstract}
Americantrypanosomiasis or Chagas disease is a neglected disease that affects about 6 to 7 million people worldwide. Its causative agent is the protozoan parasite Trypanosoma cruzi, which is transmitted to mammalian hosts by triatomine hematophagous insects. In the triatomine rectum occurs the most significant differentiation process in the parasite life cycle: the transition from replicative epimastigotes to infective trypomastigotes. In this work, a parasite intermediate form between epimastigotes and trypomastigotes was obtained by growing epimastigotes in a high osmolarity medium, mimicking partially the natural conditions found in the triatomine rectum. These intermediate forms were characterized by possessing nucleusadjacent kinetoplasts and increased flagellar length when compared to epimastigotes. Also, this transitional stage demonstrated a greater ability to interact with hydrophobic surfaces similar to those existing in the triatomine rectum. Furthermore, their microtubules presented a lower stability than those from epimastigotes, suggesting that during this differentiation process a reorganization of the micro tubular cytoskeleton takes place.
\end{abstract}

Keywords: Intermediate forms; T. cruzi ; Microtubule stability; Flagellar elongation; High osmolarity; Parasite differentiation

\section{Introduction}

Infection with the protozoan parasite Trypanosoma cruzi causes Chagas disease, a neglected disease that affects about 6 to 7 million people worldwide, mostly in Latin America (WHO, 2016). This parasite alternates between mammalian hosts and triatomine hematophagous insects, which are responsible for disease transmission. The life cycle of $T$. cruzi comprises three main forms: amastigotes, epimastigotes, and trypomastigotes, and several intermediate stages between them [1]. Due to its importance in the disease transmission, the differentiation from replicative epimastigote to the infective stage of the parasite, trypomastigote (metacyclogenesis process) has been the focus of numerous studies. In many of them the presence of intermediate forms between epi- and trypomastigotes was described [2-5]. These transitional forms described in the rectum of the insect vector, where naturally metacyclogenesis occurs, also were described in modified cultures and in the stationary phase of epimastigotes culture as a preadaptative stage for metacyclogenesis $[6,7]$. This makes these intermediate forms (IF) good candidates for study of the events that occur during the differentiation process. Some features of these intermediate forms were described by Bourguignon et al. [6], demonstrating that this transitional stage possess some biological characteristics of trypomastigotes and others of epimastigotes.

The exact events leading to the differentiation of epimastigotes are unknown so far. This process occurs in the rectum of triatomine, where increased osmolarity is caused mainly by elevated content of $\mathrm{NaCl}$ from urine. In our group, we performed experiments to find out the stress conditions in the culture medium of growing T. cruzi epimastigotes similar to those found in the rectum of triatomine bugs, by adding $0.5 \mathrm{M} \mathrm{NaCl}$ or $1 \mathrm{M}$ mannitol (which raises the osmolarity to $\sim 1,000 \mathrm{mOsm} / \mathrm{L}$ ) to the growth medium [8]. Under these conditions, we found a significant increase in the percentage of IF, constituting $90 \%$ of the parasite population at 13rd day of growth. Since $\mathrm{NaCl}$ and mannitol treatment evoked similar morphological changes, we suggested that increased osmolarity of culture medium was the factor promoting the formation of IF. In this work, we analyzed the morphological alterations that occur during this process and subsequently we studied changes in parasite's capacity to bind to hydrophobic substrates and the micro tubular rearrangements related to differentiation process.

\section{Materials and Methods}

\section{Materials}

Primary antibodies (anti-Alpha tubulin, anti-Acetylated tubulin, anti-Tyr-tubulin), secondary antibodies (anti-mouse IgG 
HRP-conjugated, anti-mouse IgG FITC- conjugated), microscope slides precoated with amino-alkyl silane, Nocodazole (methyl 5- (2- thienilcarbonil) -1H-becimidazol-2-yl) and Paclitaxel were purchased from Sigma-Aldrich. Hybond ECL nitrocellulose membranes and ECL Western Blotting detection system were purchased from Amersham (GE Healthcare).

\section{Organisms and growth conditions}

Trypanosoma cruzi Tulahuen strain was used. Epimastigote forms were grown at $28^{\circ} \mathrm{C}$ in modified Warren medium [9], supplemented with 10\% Fetal Bovine Serum (FBS), as described previously [10]. Cells in logarithmic growth phase (day 6) were harvested by centrifugation at $1,000 \mathrm{~g}$ for $10 \mathrm{~min}$, and washed twice with $25 \mathrm{mM}$ Tris- $\mathrm{HCl}, \mathrm{pH} 7.35,1.2 \mathrm{mM} \mathrm{MgSO}_{4^{\prime}}$ $2.6 \mathrm{mM} \mathrm{CaCl}, 4.8 \mathrm{mM} \mathrm{KCl}, 120 \mathrm{mM} \mathrm{NaCl}$, and $100 \mathrm{mM}$ glucose [Krebs-Ringer-Tris (KRT) buffer]. Intermediate Forms (IF) were obtained as described previously [8]. Briefly, epimastigote forms were grown in the same culture medium but in the presence of $0.5 \mathrm{M} \mathrm{NaCl}$ or $1 \mathrm{M}$ mannitol, and the cells were harvested at day 13 by centrifugation at $1,000 \mathrm{~g}$ for $10 \mathrm{~min}$ and washed twice with KRT buffer.

\section{Morphological studies}

Cells were grown as described above and fresh parasite samples were taken and quantified using a Neubauer's hemocytometric chamber. Parasites were fixed with absolute methanol, stained with $10 \%$ Giemsa in 0.1 M sodium phosphate buffer, $p H$ 7.2, and observed in a Zeiss Axiolab Microscope with a $100 \mathrm{x}$ objective. Parasites were classified as epimastigotes, trypomastigotes, or intermediate forms based on cell shape, size, and Giemsa staining, as described previously [8].

\section{Preparation of membranes}

Washed epimastigotes and IF were resuspended in five volumes of $50 \mathrm{mM}$ HEPES ( $\mathrm{pH}$ 7.4) containing $0.25 \mathrm{M}$ sucrose, $5 \mathrm{mM} \mathrm{KCl}, 1 \mathrm{mM}$ EDTA, and $4 \mu \mathrm{g} / \mathrm{ml}$ leupeptin. The suspension was frozen at $-180^{\circ} \mathrm{C}$ in liquid nitrogen and thawed; this freeze/ thaw cycle was performed three times. Membrane fractions were prepared as described previously [11]. Briefly, the homogenate was centrifuged at $100 \mathrm{~g}$ for $15 \mathrm{~min}$ to remove cell debris, and supernatant was centrifuged at $1,000 \mathrm{~g}$ for $15 \mathrm{~min}$ and at $105,000 \mathrm{~g}$ for $60 \mathrm{~min}$. Pellets from the two centrifugations were washed, re suspended with $50 \mathrm{mM}$ HEPES (pH 7.4), and used as a source of membranes. The protein content of membrane samples was determined by the Bradford method [12], with bovine serum albumin as the standard.

\section{Western blot}

50-80 $\mu \mathrm{g}$ of protein from membranes of T. cruzi epimastigotes and IF were solved in $8-12 \%$ (w/ v) SDS-polyacrylamide gel electrophoresis and electro transferred to Hybond ECL membranes. The membranes were blocked with $5 \%$ (w/v) nonfat milk suspension in PBS-Tween for $2 \mathrm{~h}$. After $1 \mathrm{~h}$ incubation with 1:1,000 dilutions of primary antibodies, detection was carried out by incubating with a 1:2,000 dilution of anti-mouse IgG HRP-conjugated. The latter was developed with the ECL Western Blotting detection system.

\section{Immuno Fluorescence Assay (IFA)}

Epimastigotes and IF were harvested and washed twice with KRT buffer as described above. Parasites were quantified using a Neubauer's hemocytometric chamber and aliquots containing $1 \times 10^{4}$ parasites were fixed in slides with methanol at $-20^{\circ} \mathrm{C}$ for 6 min, washed with PBS and incubated with PBS- $2 \%$ albumin (blocking solution) for $60 \mathrm{~min}$ at room temperature. Parasites were then incubated with 1:1,000 dilutions of primary antibodies in PBS for $60 \mathrm{~min}$, washed three times with PBS for $5 \mathrm{~min}$ and then incubated for $60 \mathrm{~min}$ with 1:2,000 dilutions of secondary antibody FITC-conjugated. Negative controls were incubated just with secondary antibody after blocking solution. The cells were observed in a Zeiss Axiolab Standard Fluorescence Microscope.

\section{Hydrophobic adhesion assay}

The assay was performed as described by Tyler and Engman [13] with some modifications. Microscope slides precoated with amino-alkyl silane were used as hydrophobic surface. Epimastigotes and IF were harvested and washed twice with KRT buffer as described above. Parasites were quantified using a Neubauer's hemocytometric chamber and aliquots containing $1 \times 10^{4}$ parasites were spread evenly over a marked $1.5 \mathrm{~cm}^{2}$ area of the slide. The parasites were allowed to adhere for $10 \mathrm{~min}$ in a humid chamber at room temperature. The slide was then washed three times in KRT for 5 min per wash, fixed in methanol at $-20^{\circ} \mathrm{C}$ for $5 \mathrm{~min}$, rehydrated in KRT for $5 \mathrm{~min}$. The slide was stained with $10 \%$ Giemsa in $0.1 \mathrm{M}$ sodium phosphate buffer, $\mathrm{pH} 7.2$ and then mounted and examined in a Zeiss Axiolab Microscope. Three independent experiments were carried out in triplicate, and attached parasites were counted in at least 10 fields by slide.

\section{Graphical and statistical analyses}

The results are shown as mean \pm standard error of the mean (SEM) for two or more independent experiments. Images were obtained and analyzed using Axio Vision 4.8 software. Quantitation and comparison of protein bands was performed using Scion Image software (an arbitrary value of 10 was assigned to the pixels $/ \mathrm{mm}^{2}$ corresponding to control samples). Graphical and statistical analysis of the data was performed using Sigma Plot 11.0 software.

\section{Results}

\section{Morphology of IF}

T. cruzi Intermediate Forms (IF) were obtained by growing of epimastigote forms in hyperosmolar media, as described previously [8]. The morphological study of these parasites was first carried out by observation (100x objectives) of preparations of Giemsa-stained samples. As shown in Figure 1, IF obtained by addition of $\mathrm{NaCl}$ (Figure 1B) or mannitol (Figure 1C) were characterized by having their kinetoplasts adjacent to the nuclei and an increase in the length of the flagellum, when compared to epimastigote forms (Figure 1A) In addition, IF possess either slender (with $\mathrm{NaCl}$ addition) or swollen (with mannitol addition) cellular bodies.

As previously reported [8], in the hyperosmotic media 
the proportion of IF increased steadily, and reaching $85-90 \%$ of parasite forms at day 13, whereas in the control culture epimastigotes remained the predominant form (65\%). The IF generated in hyperosmotic media showed normal viability, a delay in the lag period of the growth curve, increased generation time and a reduction in the maximal growth to $50 \%$ of the control value (Figure 1D). A low percentage (2-3\%) of trypomastigote forms were obtained at day 13 of growth in hyperosmolar media, similarly to observed when epimastigotes of Y Strain and Berenice Strain were incubated in saline [5].

\section{Binding to a hydrophobic substrate}

In the natural life cycle of T. cruzi, epimastigotes interact through its flagellum with triatome rectal walls, where differentiation process occurs. Such interaction, between flagellar proteins and hydrophobic substrates present in triatome rectum, would be crucial for the differentiation to trypomastigotes. Therefore, the IF's ability to interact with hydrophobic substrates was analyzed.

As hydrophobic surface, slides pre-coated with silane were used, as described by Tyler and Engman [13]. Epimastigotes and IF were allowed to interact to hydrophobic surface for 10 min and then the slides were washed and Giemsa-stained for better viewing. The samples obtained were observed by optical microscopy (Figure 2).

A total of 60 fields per condition were counted, obtaining an
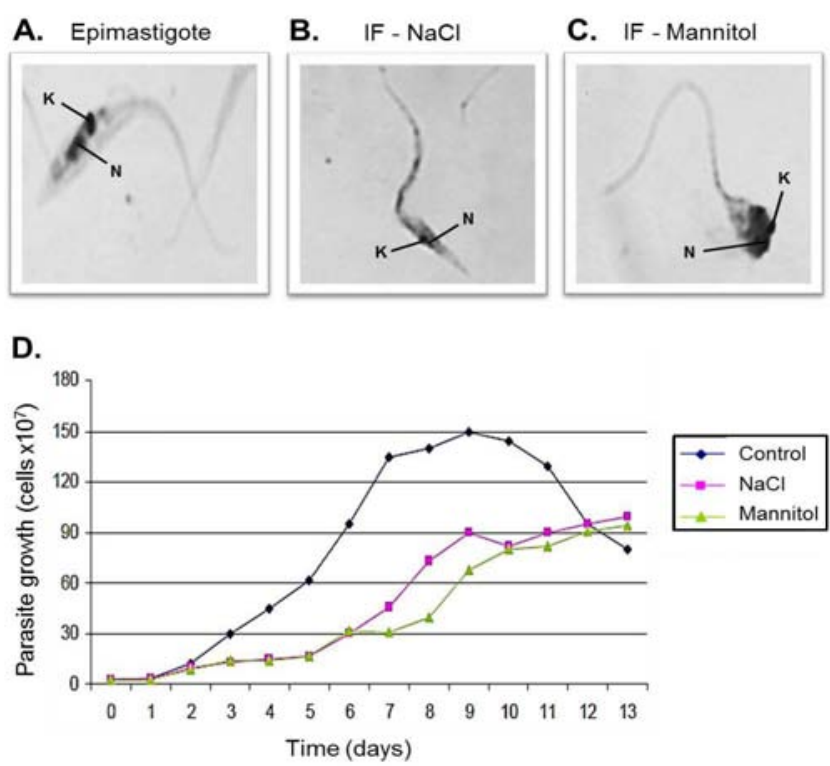

Figure 1: Trypanosoma cruzi developmental stages in axenic culture.

Parasites were fixed on slides with methanol and Giemsa-stained. Slides were observed by optical microscopy with a 100x objective. Representative images from 3 independent experiments are shown. $\mathrm{N}$ : nucleus. K: kinetoplast.
(A) Epimastigotes
(B) Intermediate forms obtained by growth with $\mathrm{NaCl}$
(C) Intermediate forms obtained by growth with mannitol
(D) Parasite growth was monitored during 13 days
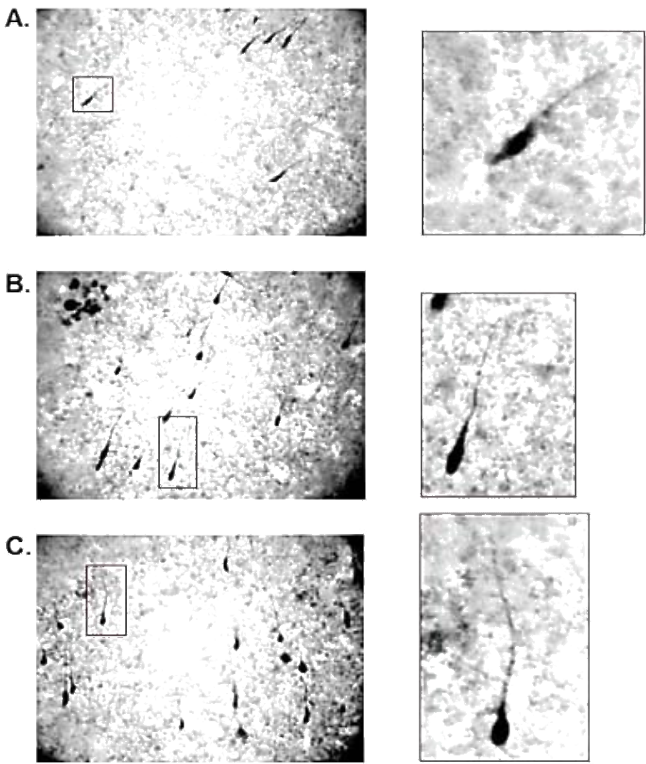

Figure 2: Hydrophobic interaction assay.

Parasites were harvested, washed and resuspended in KRT buffer as described in M\&M. 1x104 parasites were spread over a marked $1.5 \mathrm{~cm}^{2}$ area of microscope slides pre-coated with amino-alkyl silane. Interaction was allowed during $10 \mathrm{~min}$ and slides were washed, fixed with methanol and Giemsa-stained. Slides were examined by DIC microscopy (400x and 1000x). Rigth pannels: magnification of marked areas.

(A) Epimastigotes

(B) Intermediate forms obtained by growth with $\mathrm{NaCl}$

(C) Intermediate forms obtained by growth with mannitol

average of $5 \pm 3$ epimastigotes and $14 \pm 3.6$ IF adhered per field in a $40 x$ objective (Table 1 ).

In order to induce complete differentiation, parasites were allowed to adhere to hydrophobic surfaces during 10-60 min, and then were transferred to modified Grace's medium as previously described [14]. In these experimental conditions, no significant increase of trypomastigote forms was determined (not shown).

\section{Flagellar elongation measurement}

The flagellar elongation is considered an adaptation of this parasite on adverse conditions, such as nutrient deprivation [13]. As shown in the right panels of Figure 2, IF attached to hydrophobic surfaces had elongated flagella, when compared to epimastigotes. Quantitation of this difference $(P<0.05)$ is summarized in Table 1 ( $\mathrm{n}=150$ epimastigotes and $250 \mathrm{IF}$ ).

In summary, differentiation from epimastigotes to IF comprises flagellar elongation that is correlated with an increase in the parasite's ability to interact with hydrophobic substrates.

\section{Microtubule organization}

Morphological changes observed in the transition from epimastigote to intermediate form (movement of kinetoplast from pre-nuclear to adjacent position and elongation of the flagellum) should be necessarily accompanied by changes in the 
parasite microtubular network and probably by alterations in tubulin posttranslational modifications [15].

In order to study microtubular reorganization during the transition process from epimastigote to IF, the relative content of different tubulin isotypes in parasite subpellicular membranes was analyzed by Western blot. The recognition of tyrosinated tubulin (Tyr-tubulin) is indicative of microtubule dynamics, being greater the greater the presence of this subtype of tubulin. In an opposite way, stable microtubules would be enriched in acetylated tubulin [15].

Quantitative analyses were performed using Scion Image software, which determines, in pixels $/ \mathrm{mm}^{2}$, the intensity of the protein bands recognized by antibodies. For graphic plots, an arbitrary value of 10 was assigned to the number of pixels $/ \mathrm{mm}^{2}$ corresponding to the protein bands recognized in epimastigotes membranes.

The presence of tyrosinated tubulin (Tyr-tubulin) was analyzed in both stages, obtaining an increment of $60 \%$ in IF membranes, when compared with epimastigotes (Figure 3A). The presence of acetylated tubulin was also compared in both stages. This posttranslational modification occurs in a less extent in IF membranes. There were not significative differences between epimastigotes and IF membranes when total-tubulin was analyzed (Figure 3A). When analyzed by immunofluorescence assay, FITC- fluorescence (anti-acetylated tubulin) was also lower in IF, mainly in the perinuclear area (Figure 3B). As no significative differences were determined by using $\mathrm{NaCl}$ or Mannitol for obtaining IF, only the firsts are shown.

\section{Microtubule stability}

Taken together, our results indicate that during the differentiation process from epimastigotes to IF a cytoskeletal rearrangement occurs, which makes microtubules from IF less stable than those from epimastigotes. In order to confirm or refute the cytoskeleton lower stability of IF a microtubule depolymerizing agent, Nocodazole, was used.

Epimastigotes and IF were incubated with $50 \mathrm{mM}$ Nocodazole

Table 1: Morphological differences between T. cruzi epimastigotes, intermediate forms and trypomastigotes. Epimastigotes and IF obtained by growing in hyperosmotic media were morphologically analyzed. $\mathrm{N}=$ 60 fields/condition. Mean \pm SD of three independent experiments are shown.

\begin{tabular}{|c|c|c|c|}
\hline & Epimastigotes & IF & Trypomastigotes \\
\hline $\begin{array}{l}\text { Kinetoplast/ } \\
\text { Nucleus relative } \\
\text { position }\end{array}$ & Anterior & Adjacent & Posterior* \\
\hline $\begin{array}{l}\text { Flagellar length } \\
\qquad(\mu \mathrm{m})\end{array}$ & $10 \pm 1.2$ & $19 \pm 2.1^{* *}$ & $10-20^{*}$ \\
\hline $\begin{array}{l}\text { Adhered } \\
\text { parasites per } \\
\text { field }\end{array}$ & $5 \pm 3$ & $14 \pm 3.6^{* *}$ & ND \\
\hline \multicolumn{4}{|c|}{$\begin{array}{l}(*) \text { Data obtained from } \\
(* *) \text { Significative differences }(\mathrm{P}<0.05) \text { compared to epimastigotes. } \\
\text { ND: not determined. }\end{array}$} \\
\hline
\end{tabular}

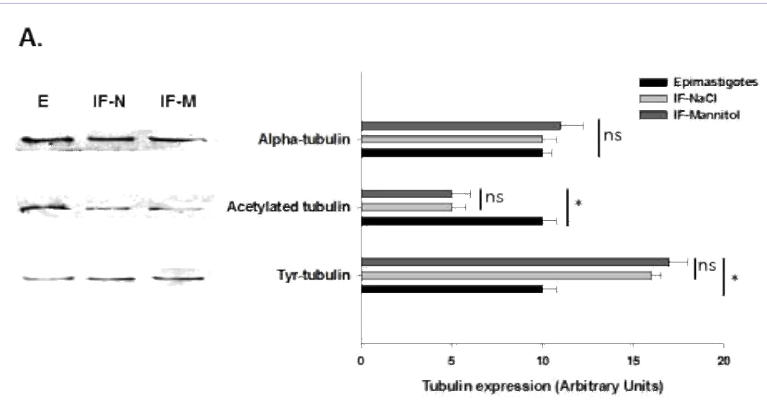

B.
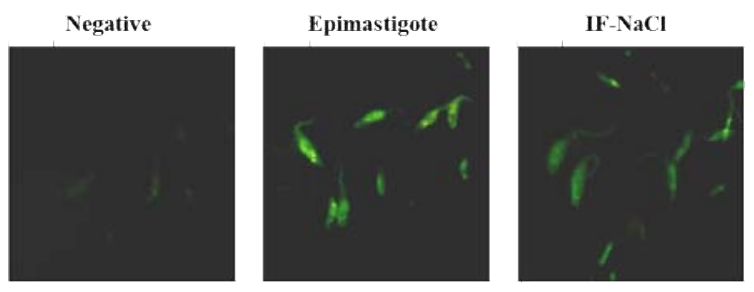

Figure 3: Tubulin posttranslational modifications in T. cruzi epimatigotes and IF.

A. Membrane fractions from epimastigotes (E) and Intermediate forms obtained by growth with $\mathrm{NaCl}$ (IF-N) or mannitol (IF-M) were analyzed by Western blot with monoclonal antibodies anti- $\propto$-tubulin, anti-acetyltubulin and anti-Tyr-tubulin. Quantitative analyses were performed using Scion Image program. Representative results from two independent experiments are shown. $\left({ }^{*}\right)$ significative differences, $\mathrm{P}<0.05$; $\mathrm{ns}=$ not significative differences.

B. Epimastigotes and Intermediate forms obtained by growth with $\mathrm{NaCl}$ (IF-NaCl) were fixed on slides and incubated with anti-acetyl-tubulin primary antibody and FITC-conjugated secondary antibody as described in M\&M. Negative control was performed by incubating parasites with secondary antibody only. Representative images from two independent experiments are shown.

during 15, 30 or 60 minutes, and subsequently membrane fractions from each stage were obtained. In these fractions, the relative amount of $\alpha$-tubulin was determined by western blot. As shown in Figure 4, microtubules from IF disassembled more quickly, suggesting less stability than those from epimastigotes. In the quantification by Scion Image program, an arbitrary value of 10 was assigned to the number of pixels $/ \mathrm{mm}^{2}$ corresponding to the protein bands obtained using both stages, un-incubated with Nocodazole (time: 0 ).

When epimastigotes and IF were incubated with 10-100 $\mu \mathrm{M}$ Paclitaxel (stabilizing microtubule agent), no significative differences in the relative amount of $\alpha$-tubulin in membrane fractions were observed (data not shown).

\section{Discussion}

The different developmental stages and differentiation processes in the life cycle of $T$. cruzi have been the focus of numerous studies, most of them emphasizing the metacyclogenesis process, the transition from replicative epimastigotes to infective trypomastigotes. Several factors have been described as involved in triggering this differentiation, such as availability of nutrients, intracellular cAMP levels and amino 


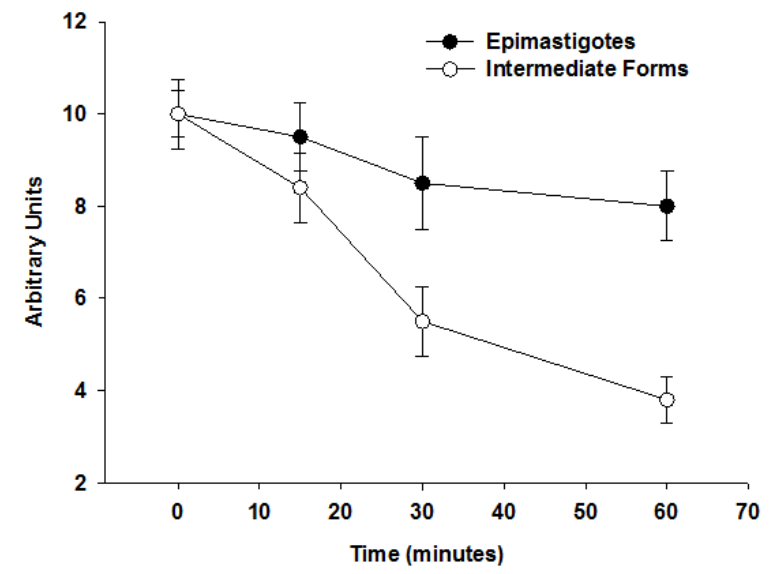

Figure 4: Comparative stability of microtubules from epimatigotes and IF. Membrane fractions from epimastigotes and Intermediate forms incubated with Nocodazole during 15, 30 or 60 minutes were analyzed by Western blot with monoclonal antibody anti- $\alpha$-tubulin. Quantification was performed with Scion Image program. An arbitrary value of 10 was assigned to the number of pixels $/ \mathrm{mm}^{2}$ corresponding to the protein bands obtained using both stages, un-incubated with Nocodazole (time: 0 ).

acid metabolism (reviewed by Goldenberg and Rodrigues Ávila, [16]) and, more recently, redox status [17]. Although it is known that metacyclogenesis can be induced in vitro by incubating epimastigotes in TAU3AAG, a chemically defined medium that mimics the urine of triatomine bugs [18], the exact events that induce this process are unknown so far.

This parasite differentiation takes place in the rectum of triatomine bugs, where epimastigotes must face an environment of high osmolarity. In this and in previous work [8] we showed that hyperosmotic conditions in the culture medium led parasites to adopt an intermediate form between epimastigote and trypomastigote, which is characterized by possessing nucleusadjacent kinetoplasts and increased flagellar length. Similarly, other authors reported that epimastigotes incubated in saline solution did not complete the differentiation process, which was interrupted in the same intermediate stage [5]. Parasite IF were also described in cultures under the same conditions in which metacyclogenesis is induced, such as nutrient limitation [13], triatomine blood ingestion [19], presence of triatomine urine [5], modification of epimastigotes culture medium [6] and epimastigotes culture aging [7]. Disregarding the method of IF generation, they share the morphological characteristics here described; i.e. elongated flagella, altered cellular body, and nucleus-adjacent kinetoplasts.

Both flagellar elongation and parasite's ability to interact with hydrophobic substrates have been considered to be a pre-adaptation of epimastigotes for differentiation to trypomastigotes [13]. It is known that the interaction of flagella with an appropriate hydrophobic substrate is a necessary event but not sufficient for differentiation [20,21]. It was suggested that the flagellar elongation occurs when epimastigotes bind to substrates similar to those existing in the triatomine rectal walls [22], and other authors also suggested that the cause of the increased hydrophobic interaction would be the limitation on glucose availability, either due to a reduction in the level of glycosylated proteins in the parasite surface or to changes in the expression of specific genes [13].

However, our results support the hypothesis of flagellar elongation as determinant factor for parasite's increased capacity of hydrophobic interaction, since IF generated by growth in high osmolarity (without changes in glucose availability) were able to adhere to hydrophobic substrates in a greater extent than epimastigotes. Several early events triggered by high osmolarity stress were determined in epimastigotes $[8,11,14]$; however their relationship with the formation of IF remains to be established.

Similarly to T. cruzi, T. brucei pre-metacyclic differentiaton involves the movement of kinetoplast, flagellar elongation and changes in the microtubule network, evidenced by tubulin posttranslational modifications $[15,23]$. The increase in the relative amount of tyrosinated tubulin in T. cruzi IF would indicate a reorganization of cytoskeleton network. This correlation was already described in T. brucei both during flagellum formation and cell division, where new microtubules intermix with pre-existing in the subpelicular cortex [24]. In addition, the reduced presence of acetylated tubulin also it involves cytoskeletal reorganization during the transition from epimastigote to IF. This is based on the general principle governing the trypanosomatids cytoskeleton: stable microtubules would be enriched in acetylated and detyrosinated tubulin [15]. This is also consistent with the fact of microtubules from IF being less resistant to a destabilizing agent as Nocodazole. Thus, we suggest that this new formation of microtubules in IF would be necessary to keep the parasite in the transitional stage, with its flagellum extended, and thus promoting the interaction with hydrophobic substrates that occurs during differentiation in the rectum of the insect vector.

\section{Acknowledgments}

This work was supported by National Agency for Scientific and Technological Promotion (ANPCyT, Argentina), Secretary of Science and Technology of the National University of Río Cuarto (SECyT, UNRC, Río Cuarto, Córdoba, Argentina) and National Council of Scientific and Technical Research (CONICET, Argentina).

\section{References}

1. Tyler KM, Engman DM. The life cycle of Trypanosoma cruzi revisited. Int J Parasitol. 2001;31(5-6):472-81.

2. Schaub GA, Losch P. Trypanosoma cruzi: origin of metacyclic trypomastigotes in the urine of the vector Triatoma infestans. Exp Parasitol. 1988;65:174-186.

3. Perlowagora-Szumlewicz A, Moreira CJ. In vivo differentiation of Trypanosoma cruzi-- 1. Experimental evidence of the influence of vector species on metacyclogenesis. Mem Inst Oswaldo Cruz. 1994;89(4):603-618.

4. Cortez MG, Gonzalez MS, Cabral MM, Garcia ES, Azambuja P. Dynamic 
development of Trypanosoma cruzi in Rhodnius prolixus: role of decapitation and ecdysone therapy. Parasitol Res. 2002;88:697-703. doi:10.1007/s00436-002-0645-0.

5. Carvalho-Moreira CJ, Spata MC, Coura JR, Garcia ES, Azambuja P, Gonzalez MS, et al. In vivo and in vitro metacyclogenesis tests of two strains of Trypanosoma cruzi in the triatomine vectors Triatoma pseudomaculata and Rhodnius neglectus: short/long-term and comparative study. Exp Parasitol. 2003;103(3-4):102-11.

6. Bourguignon SC, Mello CB, Santos DO, Gonzalez MS, Souto-Padron T. Biological aspects of the Trypanosoma cruzi (Dm28c clone) intermediate form, between epimastigote and trypomastigote, obtained in modified liver infusion tryptose (LIT) medium. Acta Trop. 2006;98(1):103-9.

7. Hernandez R, Cevallos AM, Nepomuceno-Mejia T, Lopez-Villasenor I Stationary phase in Trypanosoma cruzi epimastigotes as a preadaptive stage for metacyclogenesis. Parasitol Res. 2012;111(2):509-14. doi: 10.1007/s00436-012-2974-y.

8. Gimenez AM, Santander VS, Villasuso AL, Pasquaré SJ, Giusto NM, Machado EE. Regulation of phosphatidic acid levels in Trypanosoma cruzi. Lipids. 2011;46(10):969-79. doi: 10.1007/s11745-011-3577-6.

9. Warren LG. Metabolism of Schizotrypanum cruzi Chagas. I. Effect of culture age and substrate concentration on respiratory rate. J Parasitol. 1960;46:529-39.

10. Machado de Domenech EE, Garcia M, Garrido MN, Racagni G. Phospholipids of Trypanosoma cruzi: increase of polyphosphoinositides and phosphatidic acid after cholinergic stimulation. FEMS Microbiol Lett. 1992;74(2-3):267-70.

11. Gimenez AM, Gesumaría MC, Schoijet AC, Alonso GD, Flawiá MM, Racagni GE, etal. Phosphatidylinositol kinase activities in Trypanosoma cruzi epimastigotes. Mol Biochem Parasitol. 2015;203(1-2):14-24. doi: 10.1016/j.molbiopara.2015.10.002.

12. Bradford MM. A rapid and sensitive method for the quantitation of microgram quantities of protein utilizing the principle of protein-dye binding. Anal Biochem. 1976;72:248-54.

13. Tyler KM, Engman DM. Flagellar elongation induced by glucose limitation is preadaptive for Trypanosoma cruzi differentiation. Cell Motil Cytoskeleton. 2000;46(4):269-78.

14. Bonansea S, Usorach M, Gesumaría MC, Santander V, Gimenez AM,
Bollo M, et al. Stress response to high osmolarity in Trypanosoma cruzi epimastigotes. Arch Biochem Biophys. 2012;527(1):6-15. doi: 10.1016/j.abb.2012.07.014.

15. Gull K. The cytoskeleton of trypanosomatid parasites. Annu Rev Microbiol. 1999;53:629-55.

16. Goldenberg S, Avila AR. Aspects of Trypanosoma cruzi stage differentiation. Adv Parasitol. 2011;75:285-305. doi: 10.1016/B9780-12-385863-4.00013-7.

17. Nogueira NP, Saraiva FM, Sultano PE, Cunha PR, Laranja GA, et al. Proliferation and differentiation of Trypanosoma cruzi inside its vector have a new trigger: redox status. PLoS One. 2015;10(2):e0116712. doi: 10.1371/journal.pone.0116712.

18. Contreras VT, Salles JM, Thomas N, Morel CM, Goldenberg S. In vitro differentiation of Trypanosoma cruzi under chemically defined conditions. Mol Biochem Parasitol. 1985;16(3):315-27.

19. Kollien AH, Schaub GA. Trypanosoma cruzi in the rectum of the bug Triatoma infestans: effects of blood ingestion by the starved vector. Am J Trop Med Hyg. 1998;59(1):166-70.

20. Bonaldo MC, Souto-Padron T, de Souza W, Goldenberg S. Cellsubstrate adhesion during Trypanosoma cruzi differentiation. J Cell Biol. 1988;106(4):1349-58.

21. Kleffmann T, Schmidt J, Schaub GA. Attachment of Trypanosoma cruzi epimastigotes to hydrophobic substrates and use of this property to separate stages and promote metacyclogenesis. J Eukaryot Microbiol. 1998;45(5):548-55.

22. Kollien AH, Schmidt J, Schaub GA. Modes of association of Trypanosoma cruzi with the intestinal tract of the vector Triatoma infestans. Acta Trop. 1998;70(2):127-41.

23. Tetley L, Vickerman K. Differentiation in Trypanosoma brucei: hostparasite cell junctions and their persistence during acquisition of the variable antigen coat. J Cell Sci. 1985;74:1-19.

24. Sherwin T, Gull K. Visualization of detyrosination along single microtubules reveals novel mechanisms of assembly during cytoskeleton duplication in trypanosomes. Cell. 1989;57(2):211-21.

25. De Souza W. Basic cell biology of Trypanosoma cruzi. Curr Pharm Des. 2002;8(4):269-85. 